\title{
ANALYSE FACTORIELLE DES BIOCÉNOSES DE COLÉOPTẼRES AQUATIQUES DES MARAIS (MARISMA) DU BAS GUADALQUIVIR (ESPAGNE)
}

\author{
par A. G. Soler Andres ${ }^{1}$, C. Montes Del Olmo ${ }^{1}$ \\ et L. Ramirez Diaz ${ }^{2}$.
}

On a étudié, par des techniques d'analyses en composantes principales, les relations biocénotiques entre les différentes populations de Coléoptères aquatiques et 8 facteurs physico-chimiques du milieu. Dû à l'énorme quantité de conditions espace-temps considérées, une première analyse, portant sur 58 échantillons et 31 espèces, à montré une structure multidimensionnelle, dans laquelle il n'a pas été possible de détecter des affinités de type écologique, faunistique ou géographique. Deux analyses contenant des échantillons de milieux plus homogènes (19 échantillons et 25 espèces de marais; 15 échantillons et 23 espèces de rizière) ont permis la détermination de groupes d'espèces de distribution semblable. Les corrélations significatives entre les composantes de ces analyses et les facteurs température, $\mathrm{pH}$, chlorure, sulfate, magnésium, calcium et profondeur de l'eau, déterminent des groupes écologiques, indicateurs de certains niveaux de conditions du milieu. En plus, on observe que les variations journalières de la communauté sont plus importantes que les saisonnières.

\section{Factor analysis of the biocoenoses of aquatic Coleoptera of the marshes of lower Guadalquivir (Spain).}

Principal component analysis was used to study the biocoenotic relationships between aquatic Coleoptera and eight physical-chemical factors of the environment. Because of the large range of spatial and temporal conditions considered in this work, a preliminary analysis of 58 samples and 31 species showed a multidimensional structure in which it was not possible to detect affinities of the ecological, faunistic or geographical kind. Two analyses of samples taken from more homogenous environments (19 samples and 25 species from the marsh; 15 samples and 23 species from the rice field) revealed groups of species with a similar distribution. The significant correlations between the components of these analyses and environmental factors (temperature, $\mathbf{p H}$, chloride, sulphate, magnesium, calcium and water depth) determined the ecological groups which were indicators of certain levels of environmental conditions. Daily variations in the community were more important than seasonal changes.

1. Departamento de Zoología. Facultad de Ciencias. Universidad de Sevilla.

2. Departamento de Ecología. Facultad de Ciencias. Universidad de Sevilla 


\section{INTRODUCTION}

Les écosystèmes aquatiques du cours inférieur du Guadalquivir constituent l'un des milieux les plus intéressant du point de vue faunistique et écologique d'Europe. Cette étude a pour but fondamental de mettre en évidence les relations biocénotiques entre les différentes populations de Coléoptères aquatiques et les principales caractéristiques du milieu.

L'existence de travaux antérieurs sur la faune d'Invertébrés de cette zone (Bigot et Marazanof 1965 et 1966) schématisant de façon générale les relations de distribution de certaines espèces de Coléoptères aquatiques avec quelques facteurs abiotiques, ont servi de base à une étude plus approfondie et détaillée des variations physico-chimiques déterminant la distribution des populations dans ce milieu.

Le but de cette étude consiste à établir les principales tendances des variations des communautés en rapport avec les facteurs du milieu.

\section{MATERIEL ET MÊTHODES}

Pour l'obtention de techniques d'échantillonnage et du traitement approprié des données, on a échantillonné 28 stations parmi les différents milieux aquatiques du cours inférieur du Guadalquivir. On a identifié 55 espèces de Coléoptères aquatiques.

Les échantillonnages ont été réalisés, de façon périodique, au cours de 16 mois (de juillet 1970 à novembre 1971).

Dans le prélèvement d'échantillons des espèces, on a utilisé une passoire de $15 \mathrm{~cm}$ de diamètre, à mailles de $0,8 \mathrm{~mm}$. Pour chaque échantillon le nombre de coups de passoire dépend de la richesse totale de ce même échantillon, la valeur de chaque espèce étant obtenue en divisant le nombre d'individus par le nombre de coups de passoire. Les valeurs ainsi obtenues ont été transformées logarithmiquement $\left(\log _{10}(x+1)\right.$ (Winson et Clarke, 1940) pour leur traitement numérique postérieur.

Une description plus détaillée des caractéristiques géomorphologiques et faunistiques des milieux étudiés et des avantages de la méthode d'échantillonnage utilisée se trouve dans Soler-Andrés (1972) et Soler-Andrés et Montes (1975).

Dans les stations d'échantillonnage, on a mesuré avec la même périodicité que l'abondance d'espèces, les facteurs physico-chimiques suivants : Température de l'eau, température moyenne de l'air, $\mathrm{pH}$. ions chlorure $\left(\mathrm{Cl}^{-}\right)$, sulfate $\left(\mathrm{SO}_{4}^{=}\right)$, magnésium $\left(\mathrm{Mg}^{++}\right)$, et calcium $\left(\mathrm{Ca}^{++}\right)$et la profondeur de l'eau. Un résumé des métho- 
des employées dans leur détermination peut être consulté dans Soler-Andrés (1972).

D’une manière générale les stations d'échantillonnage peuvent être groupées dans les types d'écosystèmes suivants:

\begin{tabular}{clc}
\hline & \multicolumn{1}{c}{ Stations } & $\begin{array}{c}\text { Nombre } \\
\text { d'échantillons }\end{array}$ \\
\hline Marais & Cortijo de Cartuja & 19 \\
& Rio Guadiamar & $\mathbf{7}$ \\
\hline Lagunes & Pajas & $\mathbf{1}$ \\
d'eau douce & Santa Olalla & $\mathbf{2}$ \\
& Dulce & $\mathbf{1}$ \\
& Taraje & $\mathbf{1}$ \\
\hline & Palacio & $\mathbf{3}$ \\
\hline \multirow{2}{*}{ Caños (cours d'eau } & Raya & 1 \\
temporaires se jettant & Tio Antoñito & $\mathbf{1}$ \\
dans les marais) & Martinazo & $\mathbf{1}$ \\
& Este Guadalquivir (ebrija) & $\mathbf{1}$ \\
\hline Rizière & Cortijo de Cartuja & $\mathbf{1 5}$ \\
\hline
\end{tabular}

Pour le traitement des données on a utilisé : - le calcul des matrices de corrélation entre les abondances d'espèces et - leur analyse en composantes principales, (Harman 1967; Gittins, 1969). La rotation varimax (Harman 1967; Garcia Novo et col. 1969) qui permet une meilleure interprétation des composantes prenant en compte un faible \% de variance, a été aussi utilisée. Les nombreux avantages, que l'utilisation de ce type de technique fournit, peuvent être consultés dans Gittins (1969) et Gonzalez Bernaldez et col. (1975).

Les échantillons ont été analysés de la façon suivante :

$1^{r e}$ Analyse : Totalité des échantillons : Elle comprend 58 échantillons et 31 espèces.

$2^{e}$ Analyse : Marais du «Cortijo Cartuja»: Elle a été réalisée sur 19 échantillons et 25 espèces.

$3^{\circ}$ Analyse : Rizière du «Cortijo Cartuja»: Elle a été réalisée sur 15 échantillons et 23 espèces. 
L'objet des deux dernières analyses consiste à obtenir une précision supérieure dans la connaissance de relations, qui peuvent ne pas être décelées dans la première analyse, par suite de l'hétérogénéité du matériel considéré.

Le premier et le deuxième écosystème ne sont pas étudiés individuellement dans cette phase du travail.

\section{RESULTATS ET DISCUSSION}

\section{1. $1^{\text {re }}$ Analyse générale. Totaltté Des ÉChant Illons}

L'ensemble des 5 premières composantes n'explique pas plus de $45 \%$ de la variance totale.

Composante

I

II

III

IV

V
$\%$ variance cumulée

10,6

20,2

29,5

38,0

45,6

Ces valeurs de variance cumulée indiquent la faible efficacité de l'analyse.

Nous nous trouvons donc, dans une situation d'hypersphéricité relative, analogue à celle que d'autres auteurs, tels Margalef et Gonzalez Bernaldez (1969); Estrada (1972), ont trouvée.

Ces résultats sont expliqués par la grande amplitude de la marge de variation espace-temps des échantillons analysés, ce qui exerce une influence définitive sur les relations d'interdépendance statistique entre les variables (espèces).

Les données analysées dans ce travail présentent des relations du type suivant: Les deux espèces plus abondantes de ces marais (Noterus laevis $\delta^{*}$ et Laccophilus minutus) montrent une forte relation d'interdépendance statistique lorsqu'on étudie séparément des échantillons obtenus en deux types d'écosystème (marais et rizière) alors que cette relation devient nulle lorsqu'on étudie tous les échantillons dans l'ensemble :

\footnotetext{
* On a considéré Noterus laevis $\sigma^{*}$ et $q$ comme deux «espèces》 différentes afin de tester leurs types de comportement. Les résultats montrent que aussi bien les $\sigma^{*} \sigma^{*}$ que les ${ }^{\circ} \%$ se distribuent de la même manière. Leur relation étant à peu près, égale à 1 .
} 
Marais

Coefficient de corrélation $(* *)$ signe pour $\mathrm{p} \leqslant 0,05$.
Rizière

$+0,62^{* *}$
Totalité

d'échantillons

$+0,07$

Il est intéressant de remarquer les conséquences de ce type de phénomènes dans l'analyse factorielle, puisque le plus ou moins grand degré d'inclusion de milieux hétérogènes dans une analyse peut conduire à des conclusions très différentes concernant l'interdépendance statistique des variables (Soler-Andrés et cols., 1972).

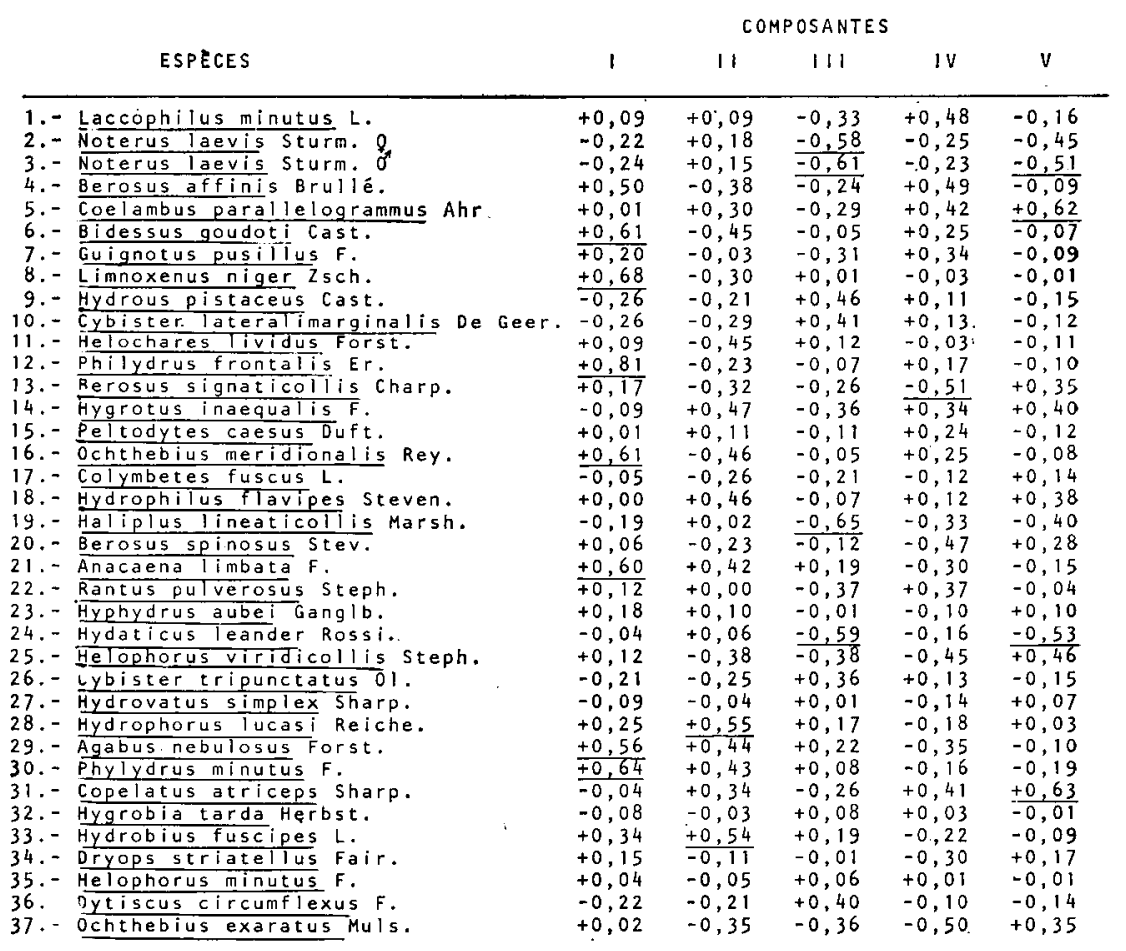

Tableau I. - Facteurs de charge non soumis à la rotation pour les cinq premières composantes de la première analyse (totalité des échantillons). On a souligné les valeurs $> \pm 0,50$.

Au tableau I sont indiqués les poids, non soumis à la rotation, des 37 espèces comprises dans l'analyse, pour les cinq premières composantes. Les facteurs soumis à la rotation n'ont pas été considérés étant donné qu'ils ne contribuaient pas à améliorer les résultats antérieurs. On a souligné les valeurs positives et négatives supérieures à 0,50 . 
Il est évident, d'après les valeurs ou contributions des espèces montrés au tableau 1, que les groupes détectés (facteurs plus élevés pour chaque composante), n'évoquent aucun type d'affinité écologique, faunistique ou géographique qui puisse les rendre facilement interprétables.

\begin{tabular}{|c|c|c|c|c|c|c|c|}
\hline & $\begin{array}{c}\mathrm{T}^{\circ} \mathrm{C} \\
\text { Moyenne } \\
\text { de l'air } \\
\end{array}$ & pH & $\mathrm{Cl} \mathrm{Na}$ & $\mathrm{SO}^{=}{ }_{4}$ & $\mathrm{Mg}^{++}$ & $\mathrm{Ca}^{++}$ & Diversité \\
\hline$\frac{\mathrm{T}^{\circ} \mathrm{C}\left(\mathrm{H}_{y} \mathrm{O}\right)}{\text { heure }}$ & 0,854 & $-0,534$ & 0,297 & $-0,045$ & 0,401 & 0,263 & 0,491 \\
\hline $\begin{array}{l}\mathrm{T}^{\circ} \mathrm{C} \text { Moyenne } \\
\text { de l'air } \\
\mathrm{pH} \\
\mathrm{Cl} \mathrm{Na} \\
\mathrm{SO}^{=} \\
\mathrm{Mg}^{++} \\
\mathrm{Ca}^{++} \\
\text {Diversité }\end{array}$ & & $-0,519$ & $\begin{array}{r}0,559 \\
-0,058\end{array}$ & $\begin{array}{r}-0,037 \\
-0,262 \\
0,277\end{array}$ & $\begin{array}{r}0,461 \\
-0,334 \\
0,534 \\
0,732\end{array}$ & $\begin{array}{r}0,218 \\
-0,758 \\
0,091 \\
0,694 \\
0,625\end{array}$ & $\begin{array}{r}0,342 \\
-0,415 \\
-0,058 \\
0,272 \\
0,472 \\
0,403\end{array}$ \\
\hline
\end{tabular}

Tablead II. - Matrice de corrélation entre les facteurs du milieux mesurés dans les échantillons de la deuxième analyse (Marais de Cortijo Cartuja). On a soutigné les valeurs significatives pour $\mathrm{p} \leqslant 0,05$.

\section{COMPOSANTES}

\begin{tabular}{|c|c|c|c|c|c|}
\hline & 1 & 11 & 111 & IV & $v$ \\
\hline 1.- Laccophilus minutus $L$ & $+0,84$ & $-0,28$ & & $+0,08$ & \\
\hline 2. - Noterus laevis Sturm. $q$ & $+0,92$ & $+0,15$ & $-0,13$ & $-0,07$ & $+0,13$ \\
\hline 3.- Noterus laevis sturm. $0^{\prime}$ & $+0,95$ & $\pm 0,06$ & $+0,02$ & $-0,10$ & $+0,08$ \\
\hline 4. - Berosus affinis Brulle: & $+0,36$ & $-0,09$ & $+0,12$ & $-0,46$ & $-0,28$ \\
\hline 5. - Coelambus parallelogrammus Ahr. & $-0,02$ & $-0,04$ & $-0,07$ & $-0,04$ & $+0,96$ \\
\hline 6. - Guignotus pusitius F. & $-0,10$ & $+0,79$ & $+0,10$ & $+0,44$ & $-0,01$ \\
\hline 7.- Limnoxenus niger Zsch. & $-0,05$ & $-0,08$ & $\pm 0,77$ & $-0,06$ & $-0,06$ \\
\hline 8. - Hydrous pistaceus Cast. & $+0,19$ & $-0,02$ & $+0,05$ & $+0,82$ & $-0,04$ \\
\hline 9. - Cybister lateralimarginalis De Geer. & $-0,30$ & $-0,56$ & & $-0,04$ & $+0,22$ \\
\hline 10. - Helochares lividus Forst. & $+0,03$ & $-0,20$ & $+0,72$ & $+0,40$ & $+0,06$ \\
\hline 11. - Philydrus frontalis Er. & $-0,28$ & $+0,31$ & $\overline{-0,11}$ & $+0,21$ & $-0,19$ \\
\hline 12.- Berosus signaticollis Charp. & $-0,13$ & $+0,54$ & $-0,17$ & -0 , & $-0,01$ \\
\hline 13.- Hygrotus inaequalis $F$. & $+0,69$ & $\overline{+0,22}$ & $-0,34$ & $+0,19$ & $-0,26$ \\
\hline 14.- Peltodytes caesus Duft. & $+0,52$ & $+0,04$ & $-0,27$ & -0 , & $-0,21$ \\
\hline 15. - Colymbetes fuscus $L$. & $+0,09$ & $-0,01$ & $+0,79$ & $-0,28$ & $-0,08$ \\
\hline 16.- Hydrophilus flavipes Steven. & $-0,08$ & $+0,70$ & $+0,33$ & $-0,03$ & $-0,02$ \\
\hline 17.- Haliplus lineaticollis Marsh. & $+0,68$ & $\overline{-0,14}$ & $-0,06$ & $+0,60$ & $+0,01$ \\
\hline 18. - Hyphydrus aubei Ganglb. & $\overline{-0,14}$ & $+0,67$ & $-0,06$ & $\overline{+0,04}$ & $-0,02$ \\
\hline 19. - Hydaticus leander Rossi. & $+0,63$ & $-0,32$ & $-0,02$ & $+0,06$ & $-0,01$ \\
\hline 20.- Helophorus viridicollis steph. & $\overline{-0,02}$ & $-0,04$ & & & $+0,96$ \\
\hline 21. - Cybister tripunctatus 01 & $-0,12$ & $-0,47$ & $+0,10$ & $+0,01$ & $-0,12$ \\
\hline 22. - Hydrovatus simplex Sharp. & $+0,08$ & $+0,04$ & $+0,87$ & $-0,12$ & $-0,01$ \\
\hline 23. - Hydroporus lucasi Reiche & $-0,15$ & $+0,56$ & $\overline{-0,18}$ & $+0,62$ & $-0,16$ \\
\hline 24. - Copelatus atriceps Sharp. & $-0,13$ & $\overline{-0,01}$ & $+0,19$ & $\overline{+0,08}$ & $-0,14$ \\
\hline 25.- Hydrobius fuscipes L. & $-0,01$ & $+0,02$ & $-0,11$ & $+0,79$ & $-0,14$ \\
\hline
\end{tabular}

Tableau III. - Facteurs de charge soumis à la rotation pour les cinq premières composantes de la deuxième analyse (Marais de Cortijo Cartuja). On a souligné les valeurs $> \pm 0,50$. 


\section{2. $2^{\circ}$ analyse. Marais du CortiJo Cartuja.}

Pour explorer de façon systématique les relations du milieu on a procédé au calcul d'une matrice de corrélation entre les facteurs mesurés dans cette station :

Température de l'eau/heure*, température moyenne de l'air, $\mathrm{pH}, \mathrm{ClNa}, \mathrm{SO}_{4}^{=}, \mathrm{Mg}^{++}, \mathrm{Ca}^{++}$et diversité (paramètre biologique).

Le tableau II indique les coefficients de corrélation entre les facteurs précédents, qui démontrent, comme l'on s'y attendait, que ces caractéristiques ne sont pas indépendantes.

Dans cette analyse, la variance cumulée pour les cinq premières composantes atteint $63,9 \%$ soit :

$\begin{array}{cc}\text { Composante } & \% \text { variance cumulée } \\ \text { I } & \mathbf{1 8 , 1} \\ \text { II } & 30,8 \\ \text { III } & \mathbf{4 2 , 9} \\ \text { IV } & \mathbf{5 4 , 9} \\ \text { V } & 63,9\end{array}$

Les facteurs non soumis à la rotation n'ont pas permis de caractériser les composantes et en conséquence, on n'étudie que ceux soumis à la rotation.

Le tableau III donne les valeurs indicatrices des espèces pour les cinq premières composantes de l'analyse.

La figure 1 représente la situation des échantillons dans le plan défini par les composantes I et II soumis à la rotation. Il est intéressant d'observer que les échantillons correspondant aux différentes heures de la journée (10 mars 1971) se groupent dans la partie supérieure du diagramme (fondamentalement à partir de la deuxième heure). Ce groupement indiquerait que la variabilité saisonnière est moins importante que la variabilité horaire (avec des changements importants de température) par rapport à la composition faunistique.

De même, les échantillons 1 et 19 (juillet 1970 et août 1971), qui correspondent aux deux échantillons de température la plus élevée se situent dans les fortes valeurs positives de la composante $\mathbf{I}$.

On a aussi examiné les relations entre les composants I, II et III soumis à la rotation et les facteurs de l'environnement (tableau IV).

* Le quotient température/heure a pour but de rendre comparables les températures prises à des heures différentes, en se basant sur l'évolution journalière type de la température dans ce volume d'eau. 


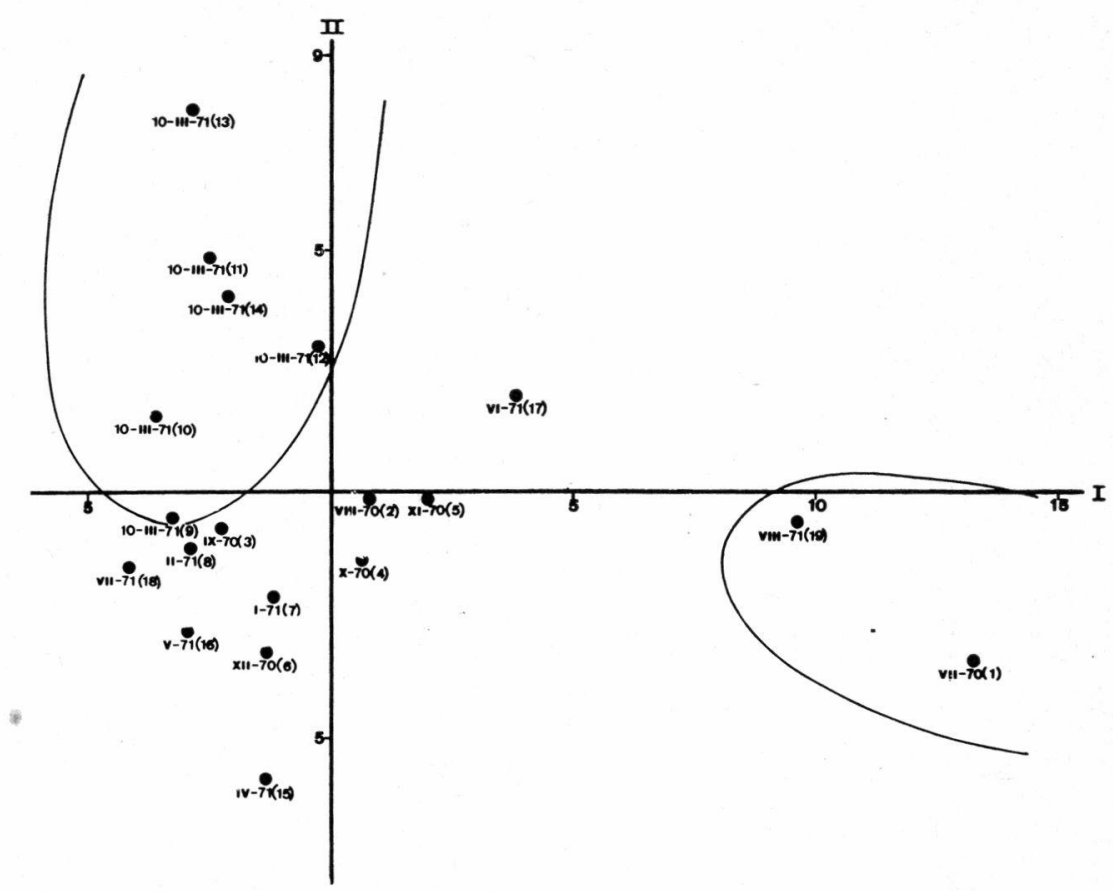

Fig. 1. - Situation des échantillons de la deuxième analyse (Marais de Cortijo Cartuja) dans le plan défini par les composantes I et II soumis à la rotation. On spécifie la date et le numéro de l'échantillon ( ) dans l'analyse pour chaque observation.

La première composante apparaît positivement corrélée avec la température moyenne de l'air et plus significativement encore avec le quotient température de l'eau/heure, et avec la diversité.

Les espèces Noterus laevis, Laccophilus minutus, Hygrotus inaequalis, Haliplus lineaticollis, Hydaticus leander et Peltodytes caesus qui possèdent des coefficients de charge élevés pour cette composante, forment donc un groupe écologique thermophile dans un certain sens, puisqu'il montre une variation d'ensemble dans le même sens que celle de la température.

La relation avec la diversité peut s'interpréter par le caractère optimum des conditions du milieu que cette composante semble caractériser. Les premières espèces mentionnées sont, en plus, les plus abondantes.

La deuxième composante montre une relation positive avec le $\mathrm{pH}$. Elle est définie par les espèces : Guignotus pusillus, Hydrophilus flavipes, Hyphydrus aubei, positivement et Cybister lateralimarginalis négativement, lesquelles possèdent des facteurs de charge élevés pour cette composante. Cette composante sépare dans sa partie positive les échantillons correspondants aux différentes heures de la même journée (23 février 1971). 
La troisième composante, matérialisée par Hydrovatus simplex, Colymbetes fuscus, Limnoxenus niger, Helochares lividus, montre un rapport négatif avec le $\mathrm{pH}$ et un rapport positif avec les concentrations de sulfate, magnésium et calcium, ainsi qu'avec la diversité.

\begin{tabular}{lrrrrrrrr}
\hline & $\mathrm{T}^{\circ} \mathrm{C}\left(\mathrm{H}_{2} \mathrm{O}\right)$ & $\begin{array}{c}\mathrm{T}^{\circ} \mathrm{C} \\
\text { Moyenne }\end{array}$ & $\mathrm{pH}$ & $\mathrm{Cl} \mathrm{Na}$ & $\mathrm{SO}^{=}{ }_{4}$ & $\mathrm{Mg}^{++}$ & $\mathrm{Ca}^{++}$ & Diversité \\
\cline { 2 - 9 } & he l'air & & & & & & \\
Composante I & 0,634 & 0,475 & $-0,408$ & $-0,043$ & $-0,118$ & 0,199 & $\mathbf{0 , 3 4 4}$ & $\mathbf{0 , 5 1 2}$ \\
Composante II & $-0,048$ & $-0,256$ & 0,500 & $-0,080$ & 0,039 & $-0,071$ & $-0,241$ & $\mathbf{0 , 1 6 6}$ \\
Composante III & 0,067 & 0,009 & $-0,467$ & 0,182 & 0,709 & 0,633 & $\mathbf{0 , 5 9 5}$ & 0,538 \\
\hline
\end{tabular}

Tableau IV. - Matrice de corrélation entre les trois premières composantes et les facteurs de milieu mesurés dans les échantillons de la deuxième analyse (Marais de Cortijo Cartuja). Sont soulignées les valeurs significatives pour $\mathrm{p} \leqslant 0,05$.

Les relations trouvées entre les composantes et quelques facteurs du milieu montrent que, dans ce cas, il est possible d'établir des «préférences» écologiques des groupes biocénotiques d'espèces corrélées.

Ces préférences apparaissent uniquement schématisées dans ce travail, mais elles sont aussi suffisamment marquées pour admettre que ces groupes d'espèces n'ont pas seulement une signification statistique (cénologique ou biocénologique) mais aussi écologique.

\section{3. $3^{\text {e }}$ analyse. Rizière du Cortijo Cartuja.}

Dans cette analyse, on a rassemblé 15 échantillons qui appartiennent, aussi dans ce cas, à un environnement relativement homogène, par rapport à l'ensemble hétérogène d'échantillons de la première analyse.

\begin{tabular}{|c|c|c|c|c|c|c|c|}
\hline & $\begin{array}{l}\mathrm{T}^{\circ} \mathrm{C} \\
\text { Moyenne } \\
\text { de l'air }\end{array}$ & $\mathrm{pH}$ & $\mathrm{Cl} \mathrm{Na}$ & $\mathrm{SO}_{4}$ & $\mathrm{Mg}^{++}$ & $\mathrm{Ca}^{++}$ & Diversité \\
\hline $\mathrm{T}^{\circ} \mathrm{C}\left(\mathrm{H}_{2} \mathrm{O}\right)$ & 0,719 & $-0,190$ & $-0,879$ & $-0,495$ & $-0,367$ & 0,026 & 0,506 \\
\hline $\begin{array}{l}\mathrm{T}^{\circ} \mathrm{C} \text { Moyenne } \\
\text { de l'air } \\
\mathrm{pH} \\
\mathrm{Cl} \mathrm{Na} \\
\mathrm{SO}^{=} \\
\mathrm{Mg}^{++} \\
\mathrm{Ca}^{++} \\
\text {Diversité }\end{array}$ & $\therefore$ & 0,234 & $\begin{array}{r}-0,706 \\
0,098\end{array}$ & $\begin{array}{r}-0,466 \\
-0,195 \\
0,679\end{array}$ & $\begin{array}{r}-0,239 \\
-0,088 \\
0,238 \\
-0,075\end{array}$ & $\begin{array}{r}-0,118 \\
-0,344 \\
0,027 \\
-0,152 \\
-0,282\end{array}$ & $\begin{array}{r}0,608 \\
0,021 \\
-0,461 \\
0,115 \\
-0,169 \\
-0,273\end{array}$ \\
\hline
\end{tabular}

Tableau V. - Matrice de corrélation entre les facteurs du milieu mesurés dans les échantillons de la troisième analyse (Rizière de Cortijo Cartuja). On a souligné les valeurs significatives pour $p \leqslant 0,05$. 
Le tableau V, montre la matrice de corrélation entre les facteurs du milieu, correspondants aux échantillons analysés. On y trouve des corrélations significatives entre la diversité et la température, et des corrélations fortement positives entre les chlorures et les sulfates. La corrélation entre la température et les chlorures et les sulfates est paradoxale. Ceci est probablement dû aux opérations d'écoulement et d'inondation, particulières à la culture du riz, signe de la nette différence entre cet environnement et le précédent (Marais du Cortijo Cartuja).

Le tableau VI montre les valeurs des poids des espèces analysées après rotation pour les cinq premières composantes.

\begin{tabular}{|c|c|c|c|c|c|c|}
\hline \multirow{2}{*}{\multicolumn{2}{|c|}{ ESPECES }} & \multicolumn{5}{|c|}{ COMPOSANTES } \\
\hline & & 1 & 11 & 111 & IV & $v$ \\
\hline $1 .-$ & Laccophilus minutus $L$. & $+0,46$ & $-0,17$ & $+0,52$ & $-0,24$ & $-0,60$ \\
\hline 2.- & Noterus laevis Sturm. of & $+0,37$ & $-0,62$ & $+0,58$ & $-0,24$ & $+0,11$ \\
\hline 3.- & Noterus laevis sturm. of & $+0,36$ & $-0,69$ & $+0,27$ & $-0,17$ & $-0,51$ \\
\hline 4.- & Berosus affinis Brullé & $-0,03$ & $-0,13$ & $-0,03$ & $-0,17$ & $-0,78$ \\
\hline 5.- & Bidessus goudoti Cast. & $+0,58$ & $+0,07$ & $.-0,16$ & $-0,02$ & $-0,60$ \\
\hline 6.- & Guignotus pusillus F. & $+0,36$ & $+0,14$ & $-0,16$ & $-0,19$ & $-0,70$ \\
\hline 7.- & Limnoxenus niger $\mathrm{zsch}$. & $+0,86$ & $+0,06$ & $-0,04$ & $-0,07$ & $-0,11$ \\
\hline 8.- & Hydrous pistaceus Cast. & $-0,13$ & $+0,22$ & $-0,11$ & $+0,80$ & $-0,02$ \\
\hline $9 .-$ & Cybister laralimarginalis De Geer. & $-0,32$ & $+0,20$ & $-0,32$ & $+0,64$ & $+0,38$ \\
\hline 10.- & Helochares lividus Forst. & $\pm 0,60$ & $-0,72$ & $+0,18$ & $-0,14$ & $-0,12$ \\
\hline $11 .-$ & Philydrus frontalis Er. & $+0,84$ & $-0,43$ & $-0,11$ & $-0,09$ & $-0,22$ \\
\hline $12 .-$ & Hygrotus inaequalis $F$. & $+0,10$ & $-0,95$ & $-0,03$ & $-0,10$ & $-0,10$ \\
\hline 13.- & Peltodytes caesus Duft. & $+0,89$ & $-0,08$ & $+0,13$ & $-0,13$ & $-0,27$ \\
\hline $14 .-$ & Colymbetes fuscus L. & $-0,27$ & 0,27 & $-0,08$ & $+0,29$ & $+0,05$ \\
\hline 15.- & Hydrophlius flavipes Steven. & $-0,05$ & $+0,25$ & $-0,23$ & $-0,45$ & $+0,39$ \\
\hline 16. - & Haliplus lineaticollis Marsh. & $-0,10$ & $-0,93$ & $-0,11$ & $-0,07$ & $+0,10$ \\
\hline 17.- & Anacaena limbata $\mathrm{F}$ & $-0,14$ & $+0,26$ & $+0,95$ & $-0,07$ & $-0,04$ \\
\hline $18 .-$ & Rantus pulverosus Steph. & $-0,04$ & $+0,06$ & $+0,92$ & $-0,07$ & $+0,13$ \\
\hline 19.- & Hydaticus leander Rossi. & $+0,43$ & $+0,14$ & $+0,58$ & $-0,32$ & $+0,53$ \\
\hline $20 .-$ & Cybister tripunctatus 01 . & $-0,15$ & $+0,09$ & $-0,16$ & $+0,84$ & $+0,24$ \\
\hline $21 .-$ & Hygrobia tarda Herbst. & $+0,29$ & $+0,1 \mathrm{I}$ & $-0,03$ & $-0,05$ & $\underline{-0,75}$ \\
\hline 22.- & Hydrobius fuscipes L. & $-0,05$ & $-0,97$ & $-0,06$ & $-0,06$ & $+0,06$ \\
\hline 23.- & Dytiscus circumflexus $F$. & $+0,03$ & $+0,10$ & $-0,01$ & $+0,83$ & $+0,12$ \\
\hline
\end{tabular}

Tableau VI. - Facteurs de charge soumis à la rotation pour les cinq premières composantes de la troisième analyse (Rizière de Cortijo Cartuja). On a souligné les valeurs $> \pm 0,50$. 



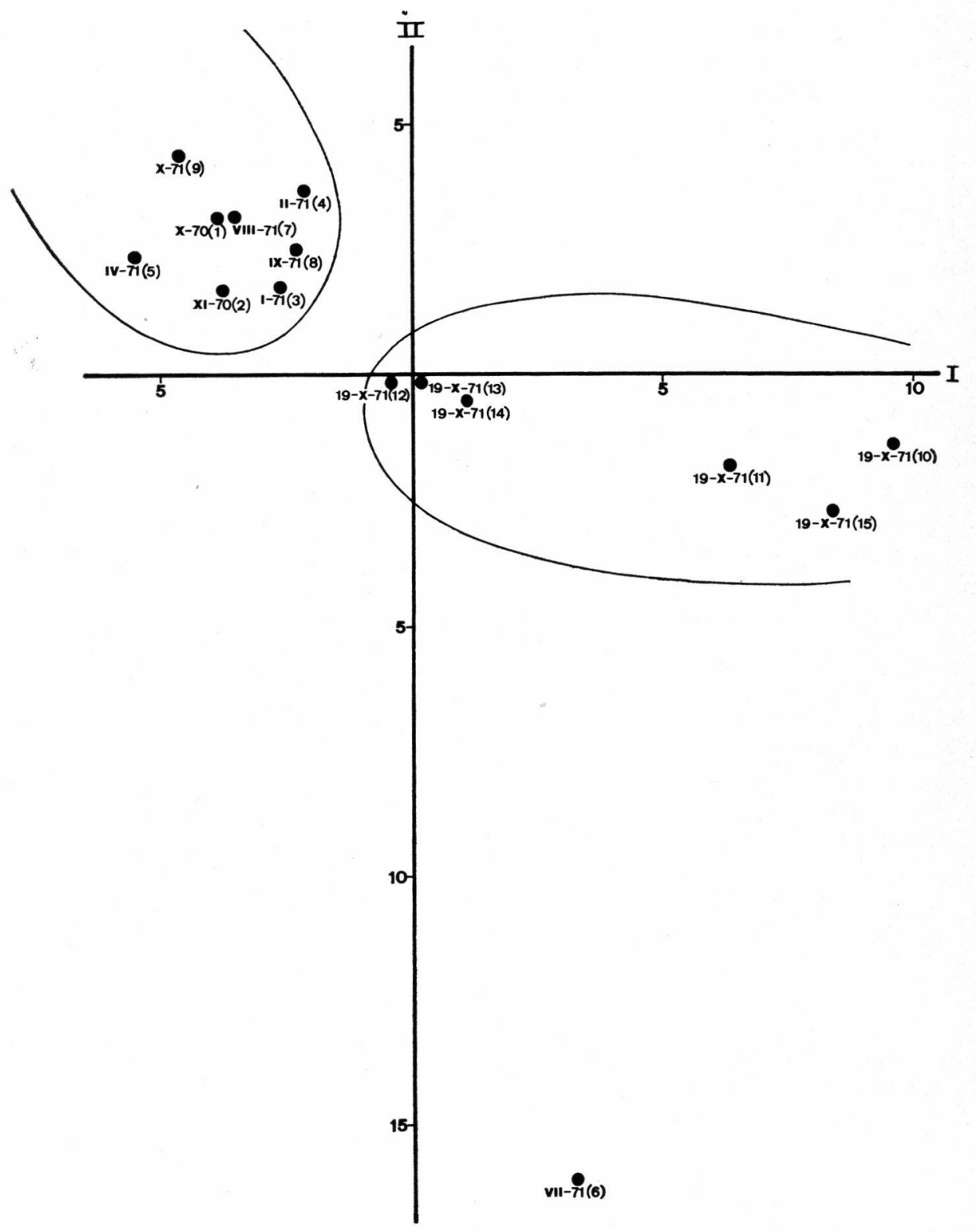

FIG. 2. - Situation des échantillons dans la troisième analyse (Rizière de Cortijo Cartuja) dans le plan défini par les composantes I et II soumis à la rotation. Mêmes symboles que dans la figure 1 . 


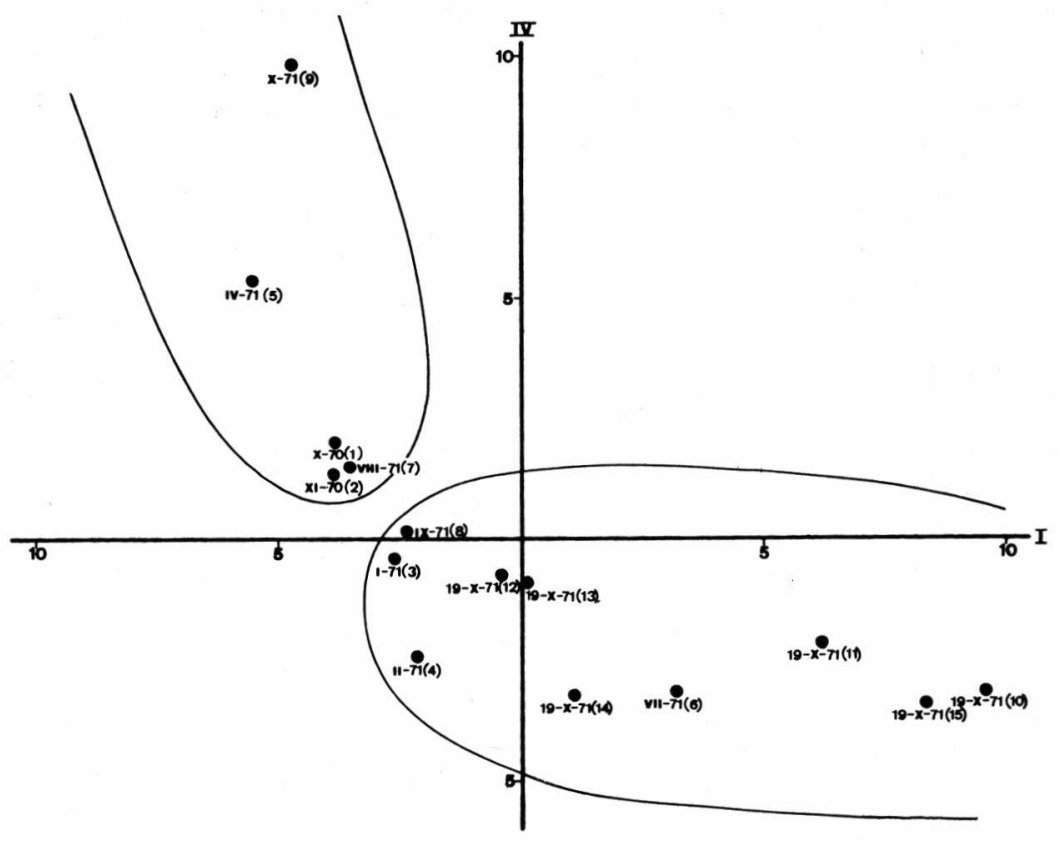

FIg. 3. - Situation des échantillons dans la troisième analyse (Rizière de Cortijo Cartuja) dans le plan défini par les composantes I et IV soumis à la rotation. Mêmes symboles que dans les figures 1 et 2 .

Le groupe d'espèces indicatrices de la composante IV attire l'attention; il est formé par les espèces de plus grande taille et par celles de hauts niveaux de l'eau : Cybister tripunctatus, Dytiscus circumflexux, Hydrous pistaceus et Cybister lateralimarginalis. Ceci correspond à de hauts niveaux d'eau pendant l'échantillonnage. Le coefficient de cette composante avec la profondeur de l'eau dans les différents échantillons est de $+0,878$ (signe pour $\mathrm{p} \leqslant 0,01$ ).

\section{CONCLUSIONS}

La grande quantité de conditions espace-temps considérées (multiplicité d'habitats et de dates d'échantillonnage) lors de l'inclusion de tous les échantillons dans une seule analyse factorielle, se traduit par des résultats peu efficaces. Il n'y a pas, donc, de directions privilégiées de variation et, par conséquent, des affinités de type écologique, faunistique ou géographique ne sont pas décelables. 
Lorsqu'on n'étudie que des échantillons qui appartiennent à des milieux homogènes, la fraction de variance expliquée dans chaque analyse augmente quand la variation espace-temps du matériel analysé diminue.

Cette augmentation de l'homogénéité se traduit dans la détection de groupes d'espèces, caractérisés par des coefficients de charge élevés * (soulignés sur les tableaux III et VI) et qui peuvent être considérés commme des groupes cénologiques (groupes d'espèces de distribution semblable).

L'obtention de corrélation significatives entre les composantes qui représentent les variations d'abondance de tels groupes d'espèces, et les facteurs physico-chimiques du milieu, permet de définir des groupes écologiques, indicateurs de certains niveaux de conditions de l'environnement. On peut, entre autres, citer les groupes suivants :

\begin{tabular}{|c|c|c|c|}
\hline Ecosystème & Espèces & Facteur & $\begin{array}{l}\text { Signe } \\
\text { de la } \\
\text { corrélation }\end{array}$ \\
\hline $\begin{array}{l}\text { Marais } \\
\text { et } \\
\text { rizière }\end{array}$ & $\begin{array}{l}\text { Noterus laevis } \\
\text { Laccophilus minutus } \\
\text { Haliplus lineaticollis } \\
\text { Helochares lividus }\end{array}$ & $\begin{array}{l}\text { Température } \\
\text { Diversité }\end{array}$ & $\begin{array}{l}+ \\
+\end{array}$ \\
\hline Marais & $\begin{array}{l}\text { Hydrovatus simplex } \\
\text { Colymbetes fuscus } \\
\text { Limnoxenus niger }\end{array}$ & $\begin{array}{l}\mathrm{pH} \\
\mathrm{SO}_{4}^{=}, \mathrm{Mg}^{++}, \mathrm{Ca}^{++}\end{array}$ & $\begin{array}{l}+ \\
-\end{array}$ \\
\hline Rizière & $\begin{array}{l}\text { Cybister tripunctatus } \\
\text { Dytiscus circumflexus } \\
\text { Hydrous pistaceus } \\
\text { Cybister lateralimarginalis }\end{array}$ & $\begin{array}{l}\text { Profondeur } \\
\text { de I'eau }\end{array}$ & + \\
\hline
\end{tabular}

Les fluctuations de la composition de la communauté, dans les échantillons pris tout au long d'une même journée (forte variation de température), sont plus importantes que les variations saisonnières. Dans l'ordination d'échantillons fournie par les analyses de marais et rizière, les échantillons d'une même journée apparaissent groupés (coordonnées semblables dans les composantes).

\footnotetext{
* Chiffres qui expriment l'affinité pour chaque composante ou direction plus importante de la variation biocénotique.
} 


\section{TRAVADX CITES}

Bigot (L.) et Marazanof (F.). 1965. - Considérations sur l'écologie des (Invertébrés terrestres et aquatiques des Marismas du Guadalquivir (Andalucia). Vie et milieu, $16: 441-473$.

Bigot (L.) et Marazanof (F.). 1966. - Notes sur l'écologie des Coléoptères aquatiques des Marismas du Guadalquivir et premier inventaire des Coléoptères et Lépidoptères du Coto Doñana (Andalucía). Annls Limnol., 2 : 491-502.

Estrada (M.). 1972. - Analyse en Composantes principales de données de phytoplancton de la zone côtière du Sud de l'Ebre.lnv. Pesq., 36 (1) : 109-118.

Garcia Novo (F.), Gonzalez Bernaldez (F.) et Gil Criado (A.). 1969. Essai d'analyse automatique de la végétation et des facteurs du milieu. V'Symposium Flora Europaea. Publ. Univ. Sevilla, Sevilla.

Gitrins (R.). 1969. - The application of ordination techniques. In : I. H. Rorison, Ecological aspects of the mineral nutrition of plants. Blackwell, Oxford.

Gonzalez Bernatdez (F.), Garcia Novo (F.) et Ramirez Diaz (L.). 1975. - Analyse factorielle de la végétation des dunes de la réserve Biologique de Doñana (Espagne). I. Analyse des données floristiques. Israel J. a Botany, $24: 106-117$.

Harman (H. H.). 1967. - Modern factor analysis. University of Chicago Press, Chicago.

Margatef (R.) y Gonzalez Bernaldez (F.). 1969. - Grupos de especies asociadas en el fitoplancton del Mar Caribe (NE de Venezuela). Inv. Pesq., 33 : 287-312.

Soler Andres (A.), Gonzalez Bernaldez (F.), Ramirez Diaz (L.) y GaRcia Novo (F.). 1972. - Variaciones de las correlaciones interespecíficas en diferentes medios. Su influencia en el análisis factorial de biocenosis, Inv. Pesq., 36 (1) : 141-144.

Soler Andres (A.). 1972. Los Coleópteros Acuáticos de las Marismas del Bajo Guadalquivir. Serv. Publ. Univ. Sevilla, Sevilla.

Soler Andres (A.) y Montes Des. Olmo (C.). 1975. - Datos para el conocimiento de los Coléopteros acuáticos de la cuenca del rio Guadalquivir. HI. Marisma del Bajo Guadalquivir (Estudio faunístico y zoogeografico (en preparacion).

Winson (C. P.) and Clarke (G. L.). 1940. - A statistical study of variation in the Catch of plankton nets. J. mar. Res., $3: 1-34$. 